\title{
Genome editing in avian research- an update review
}

\author{
N.H.Tinh ${ }^{1}$, A.P.N. Bui ${ }^{2 *}$
}

${ }^{1}$ Institute of Animal Sciences for Southern Vietnam, Hiep Thang quarter, Binh Thang ward, Di An town, Binh Duong city, ${ }^{2}$ Faculty of Biotechnology, Ho Chi Minh city Open University, 35 Ho Hao Hon street, Ho Chi Minh city, Vietnam

"Corresponding author: Tel 0084-8-39300086; Email: buiphunamanh@ yahoo.com

Journal of Livestock Science (ISSN online 2277-6214) 11: 139-142

Received on 2/7/2020; Accepted on 17/9/2020

doi. 10.33259/JLivestSci.2020.139-142

\begin{abstract}
Chicken contributes significant portion in human daily meals and serves as a research model in genome editing. As a result, research in elucidating gene functions and directional modification of the phenotypes aimed for customerdriven needs are highly emphasized in broiler industry. The development of genome editing technologies including zinc finger nucleases (ZFNs), transcription activator-like effector nucleases (TALENs) and the latest cluster regularly interspaced short palindromic repeat (CRISPR)/Cas9 (CRISPR/Cas9) revolutionized the understandings in chicken development and offered novel tools in generating genome edited chicken to improve chicken productivity and health. This review will provide the principles of genome editing techniques and latest updates of their applications in improving chicken productivity and health.
\end{abstract}

Keywords: CRISPR/Cas9; genome editing; animal welfare; productivity; performance. 
Tinh and Bui, 2020/J. Livestock Sci. 11: 139-142

\section{Introduction}

Agriculturists have applied selective breeding to ameliorate traits of great economic importance for thousands of years. In broiler industry, genetically specialized characteristics such as meat yield, feed efficiency, fat content in males, and fertility, rate of egg production, hatchability in females, are highly favored (Qanbari, et al. 2019; Hartcher and Lum 2020). Although through artificial selection, creation of new breeds with better performance was obtained, this generating process was slow and time-consuming (Fernyhough, et al. 2020). In the late $20^{\text {th }}$ century, with the employment of genomic selection, certain desirable phenotypic features were assigned to specific genomic locations, thus faster enhancement in broiler productivity has been achieved (Proudfoot, et al. 2019). However, there are several obstacles in measuring inherently difficult quantifiable phenotypes such as disease resistance, or welfare-related traits in avian research. In disease resistance, as animals with less severe symptoms could have been exposed to less pathogen, maintaining equal pathogenic exposure to large numbers of animals for genetic linkage mapping is expensive and laborious. In welfare-related traits such as bone strength, characterizing the femur elements requires animal sacrifice, making it ethically questionable (Boodhoo, et al. 2016; Guo, et al. 2019; Proudfoot, et al. 2019). The advent of genome editing provides new opportunities for rapid and direct translation from laboratory research to farm-set broiler production.

\section{Genome editing and its principle \\ 1. ZFN and TALEN systems}

Genome editing techniques employ programable nucleases to introduce genetic mutation (Laible 2018; McFarlane, et al. 2019). Early gene editing systems including ZFN and TALEN depend on the specificity of the DNA-binding domain of zinc finger protein (ZFP) in the ZFN system and transcription activator-like effector (TALE) in the TALEN system. Since each zinc finger in the ZFP recognizes every triplet on single-strand DNA, designing 3-6 zinc finger components in combination will therefore attach to 9-18 base pairs on aimed regions to achieve specificity. On the other hand, the improved targeting property of TALE relies on the programmable tandem repeat modules, of which each module specifically binds to a single base pair (Tait-Burkard, et al. 2018). The order of the tandem repeat modules can be rearranged to obtain better directing at chosen DNA sequence. After the binding to DNA region, both ZFP and TALE will orchestrate the dimerized endonuclease Fok 1 to break the double strand DNA at predetermined regions (Carlson, et al. 2012). The application of ZFN and TALEN have been widely applied in pig and cattle breeding (Yum, et al. 2018; Wu and Bazer 2019).

\section{CRISPR/Cas9 system}

The latest CRISPR/Cas9 system was firstly identified as an adaptive defense barrier in bacteria. The system is made up of two components including the CRISPR locus and Cas9 endonuclease (Miao, et al. 2019). Upon infection, trans-activated RNA (tracrRNA) is transcribed from the CRISPR locus and subsequently joined crRNA and Cas9 protein to become effector complex. Since tracrRNA is the transcription of the past invading foreign nucleic acid, it will have the matching sequences to the exogenous DNA (Wu and Bazer 2019). CrRNA will direct the Cas9 protein to bind and degrade the foreign DNA. Recently, CRISPR/Cas9 system has been refined and optimized for genome editing purpose in eukaryotes. The tracrRNA and the crRNA will be integrated to become single guide RNA (sgRNA) which can be programmable to specifically target any desirable sequences by production of mutations or insertion of transgenes (Kaulich and Dowdy 2015; Wang, et al. 2015).

The introduction of DSB generated by three genome editors including ZFNs, TALENs and CRISPR/Cas9 will trigger the DNA repair mechanisms including non-homologous end-joining (NHEJ) or homologous recombination (HR) (Gupta and Musunuru 2014). In the error prone NHEJ pathway, the two ends of the cleaved DNA are joined and ligated, resulting in the generation of insertion or deletion at the site of DSB, thus producing knock-out mutation (Kaulich and Dowdy 2015). In the HE pathway, a site-directed nuclease and an exogenous DNA template harboring homologous sequence to the DSB regions are required to facilitate the insertion of single or multiple transgenes, thereby gaining knock-in mutation (Wang, et al. 2015). In comparison with the previous genome editing techniques including ZFN and TALEN, CRISPR/Cas9 system is easier to design and cheaper to apply in research (Liu, et al. 2017).

\section{Generation of edited chickens}

Due to the physiological differences between avian egg and mammalian oocyte, delivery of editing reagents into the avian zygote pose challenges in the creation of genome edited chickens (Mozdziak and Petitte 2004). One study performed in 2017 has suggested in ovo electroporation of CRISPR/Cas9 components, which enabled the analysis of the knock-down genes in the neural crest (Gandhi, et al. 2017). However, electroporation can produce mosaicism since the avian embryo has been further established when an egg is laid compared to the mammalian zygote (Véron, et al. 2015). Alternative approach is sperm transfection-assisted gene editing whereby 
sperm are lipofected with editing reagents prior to artificial insemination (Cooper, et al. 2017). Thanks to the latest breakthrough in chicken stem cell technology, primordial germ cells (PGCs) are proposed to be promising resources for genome edition (Sid and Schusser 2018). Since PGCs are highly specialized cells and precursors of sperm, they can be collected from the blood of the developing chicks in ovo and in vitro cultured. After being genome edited, PGCs are transferred back into the bloodstream of the stage-matched recipients. Subsequently, these edited germ cell lines will migrate to the gonad and can be utilized in breeding purposes (Cooper, et al. 2018). The main advantages of PGCs employment for genome editing over previous methods are the reliability of germline transmission, low number of animals used in the experiment, thus ideal for generating gene-edited chickens (Wang, et al. 2020). However, many drawbacks have been associated with PGCs such as maintaining germline competency, low efficiency and persistence in transfection, and quiescent transcription of germ cell lines. Accumulation of efforts have been done to optimize the PGCs-mediate germline transmission system for establishment of genome-edited poultry breeding (Sid and Schusser 2018).

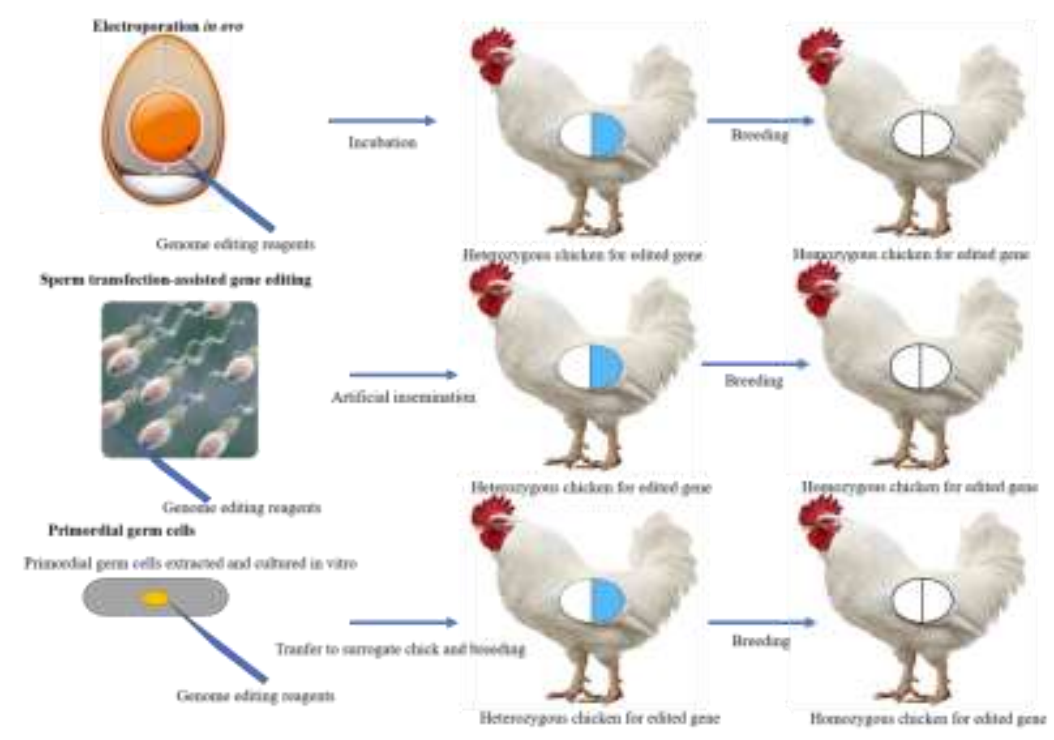

Fig 1. Methods for delivery of editing reagents into the avian zygote pose challenges in the creation of genome edited chickens

\section{Application of genome editing in broiler industry}

Due to the unique structure of the avian embryos compared to the mammalian embryos, a small number of research have been reported on the generation of genome-edited chickens for agricultural purposes. Creating ZFNmediated gene editing chicken is not yet documented, and there have been only limited results on utilizing TALEN and CRISPR/Cas9 systems in chicken. In 2014, ovalbumin (OVA) gene was targeted to produce OVA knock out chickens by transfecting cultured PGCs with plasmid encoding OVA-TALEN (Park, et al. 2014). More recently, by combining TALEN and homology directed repair, Taylor et al. (2017) have obtained sterile hens by disrupting DD4X locus which is allegedly responsible for germ line formation (Taylor, et al. 2017). In 2018, in attempt to achieve disease-resistant chicken, the avian leukosis virus (ALV) receptor was edited in chicken somatic cell lines by CRISPR/Cas9 system (Koslová, et al. 2018). It is hoped that these findings will shed new lights in applying genome editing to improve important agricultural traits in broiler industry.

\section{References}

1) Boodhoo N, Gurung A, Sharif S, Behboudi S. 2016. Marek's disease in chickens: a review with focus on immunology. Veterinary Research 47:119.

2) Carlson DF, Tan W, Lillico SG, Stverakova D, Proudfoot C, Christian M, Voytas DF, Long CR, Whitelaw CBA, Fahrenkrug SC. 2012. Efficient TALEN-mediated gene knockout in livestock. Proceedings of the National Academy of Sciences of the United States of America 109:17382-17387. 
3) Cooper CA, Challagulla A, Jenkins KA, Wise TG, O’Neil TE, Morris KR, Tizard ML, Doran TJ. 2017. Generation of gene edited birds in one generation using sperm transfection assisted gene editing (STAGE). Transgenic Research 26:331-347.

4) Cooper CA, Doran TJ, Challagulla A, Tizard MLV, Jenkins KA. 2018. Innovative approaches to genome editing in avian species. Journal of animal science and biotechnology 9:15-15.

5) Fernyhough M, Nicol CJ, van de Braak T, Toscano MJ, Tønnessen M. 2020. The Ethics of Laying Hen Genetics. Journal of Agricultural and Environmental Ethics 33:15-36.

6) Gandhi S, Piacentino ML, Vieceli FM, Bronner ME. 2017. Optimization of CRISPR/Cas9 genome editing for loss-of-function in the early chick embryo. Developmental biology 432:86-97.

7) Guo Y, Tang H, Wang X, Li W, Wang Y, Yan F, Kang X, Li Z, Han R. 2019. Clinical assessment of growth performance, bone morphometry, bone quality, and serum indicators in broilers affected by valgus-varus deformity. Poultry Science 98:4433-4440.

8) Gupta RM, Musunuru K. 2014. Expanding the genetic editing tool kit: ZFNs, TALENs, and CRISPR-Cas9. The Journal of clinical investigation 124:4154-4161.

9) Hartcher KM, Lum HK. 2020. Genetic selection of broilers and welfare consequences: a review. World's Poultry Science Journal 76:154-167.

10) Kaulich M, Dowdy SF. 2015. Combining CRISPR/Cas9 and rAAV Templates for Efficient Gene Editing. Nucleic Acid Therapeutics 25:287-296.

11) Koslová A, Kučerová D, Reinišová M, Geryk J, Trefil P, Hejnar J. 2018. Genetic Resistance to Avian Leukosis Viruses Induced by CRISPR/Cas9 Editing of Specific Receptor Genes in Chicken Cells. Viruses 10:605.

12) Laible G. 2018. Production of Transgenic Livestock: Overview of Transgenic Technologies. In: Niemann H, Wrenzycki C, editors. Animal Biotechnology 2: Emerging Breeding Technologies. Cham: Springer International Publishing. p. 95-121.

13) Liu C, Zhang L, Liu H, Cheng K. 2017. Delivery strategies of the CRISPR-Cas9 gene-editing system for therapeutic applications. Journal of controlled release : official journal of the Controlled Release Society 266:17-26.

14) McFarlane GR, Salvesen HA, Sternberg A, Lillico SG. 2019. On-Farm Livestock Genome Editing Using Cutting Edge Reproductive Technologies. Frontiers in Sustainable Food Systems 3.

15). Miao D, Giassetti MI,Ciccarelli M, Lopez-Biladeau B,Oatley JM. 2019. Simplified pipelines for genetic engineering of mammalian embryos by CRISPR-Cas9 electroporation. Biology of Reproduction 101:177-187.

16) Mozdziak PE, Petitte JN. 2004. Status of transgenic chicken models for developmental biology. Developmental Dynamics 229:414-421.

17) Park TS, Lee HJ, Kim KH, Kim J-S, Han JY. 2014. Targeted gene knockout in chickens mediated by TALENs. Proceedings of the National Academy of Sciences of the United States of America 111:12716-12721.

18) Proudfoot C, Lillico S, Tait-Burkard C. 2019. Genome editing for disease resistance in pigs and chickens. Animal Frontiers 9:6-12.

19) Qanbari S, Rubin C-J, Maqbool K, Weigend S, Weigend A, Geibel J, Kerje S, Wurmser C, Peterson AT, Brisbin IL, Jr., Preisinger R, Fries R, Simianer H, Andersson L. 2019. Genetics of adaptation in modern chicken. PLOS Genetics 15:e1007989.

20) Sid H, Schusser B. 2018. Applications of Gene Editing in Chickens: A New Era Is on the Horizon. Frontiers in genetics 9:456-456.

21) Tait-Burkard C, Doeschl-Wilson A, McGrew MJ, Archibald AL, Sang HM, Houston RD, Whitelaw CB, Watson M. 2018. Livestock 2.0 - genome editing for fitter, healthier, and more productive farmed animals. Genome Biology 19:204.

22) Taylor L, Carlson DF, Nandi S, Sherman A, Fahrenkrug SC, McGrew MJ. 2017. Efficient TALEN-mediated gene targeting of chicken primordial germ cells. Development (Cambridge, England) 144:928-934.

23) Véron N, Qu Z, Kipen PAS, Hirst CE, Marcelle C. 2015. CRISPR mediated somatic cell genome engineering in the chicken. Developmental biology 407:68-74.

24) Wang K, Ouyang H, Xie Z, Yao C, Guo N, Li M, Jiao H, Pang D. 2015. Efficient Generation of Myostatin Mutations in Pigs Using the CRISPR/Cas9 System. Scientific reports 5:16623-16623.

25) Wang X, Shields LE, Welch RL, Pigg A, Kaleh K. 2020. Chapter 10 - Transgenesis and genome editing in chickens. In: Malik YS, Barh D, Azevedo V, Khurana SMP, editors. Genomics and Biotechnological Advances in Veterinary, Poultry, and Fisheries: Academic Press. p. 223-247.

26) Wu G, Bazer FW. 2019. Application of new biotechnologies for improvements in swine nutrition and pork production. Journal of animal science and biotechnology 10:28.

27) Yum S-Y, Youn K-Y, Choi W-J, Jang G. 2018. Development of genome engineering technologies in cattle: from random to specific. Journal of animal science and biotechnology 9:16-16. 